\title{
GNC requirements of for a Long Range Hypersonic Transport System
}

\author{
Rodrigo Haya-Ramos ${ }^{1}$, Gabriele de Zaiacomo ${ }^{2}$, Jordi Freixa ${ }^{3}$ \\ Deimos Space S.L.U., Madrid, 28760, Spain \\ Martin Sippel ${ }^{4}$ \\ German Aerospace Center (DLR), Space Launcher Systems Analysis (SART), Bremen, 28359, Germany \\ Rafael Molina ${ }^{5}$ \\ European Space Agency, ESTEC, Noordwijk, The Netherlands
}

The objective of a long range hypersonic transportation system is to take advantage of high altitude and high velocity to perform intercontinental flight in the order on one hour. The SpaceLiner concept has been proposed by the Space Launcher Systems Analysis group (SART) of the German Aerospace Center (DLR). In the frame of the European Commission project FAST20XX, this concept has been further investigated in several areas. This paper presents the results of the Guidance, Navigation and Control (GNC) analyses for the SpaceLiner concept. The flight mechanics of the Spaceliner concept is analysed to provide requirements in terms of Flying Qualities, layout (centre of gravity location), angle of attack corridor and entry corridor during the flight. Trajectory control capability is assessed through Guidance assessments. An adaptive guidance technique has been layout to assess the trajectory control margins and expected guidance performance. Alternative flight trajectories have been proposed to ensure margins for trajectory control while preserving the main vehicle and mission constraints.

\section{Nomenclature}

AoA, $\alpha=$ Angle of Attack

$\mathrm{C}_{\mathrm{mcg} \alpha}=$ Pitch moment coefficient derivative with respect to the angle of attack at the CoG

$\mathrm{CL} \quad=$ Lift Coefficient

$\mathrm{CD} \quad=$ Draft Coefficient

$\mathrm{CDE}=$ Draft Coefficient due to elevator deflection

$\mathrm{CDBF}=$ Draft Coefficient due to body flap deflection

$\mathrm{CM} \quad=$ Pitch moment coefficient

$\mathrm{d}=$ Drag acceleration

$\delta_{\mathrm{e}} \quad=$ Elevator deflection

$\delta_{\mathrm{bf}} \quad=$ Body flap deflection

$\mathrm{L} / \mathrm{D} \quad=$ Lift to drag ratio

$\mathrm{M} \quad=$ Mach number

\footnotetext{
${ }^{1}$ Head of Atmospheric Flight CC. Aerospace Engineering BU, rodrigo.haya@ deimos-space.com, AIAA member

${ }^{2}$ Atmospheric Flight CC, gabriele.dezaiacomo@deimos-space.com.

${ }^{3}$ Atmospheric Flight CC, jorge.serna@ deimos-space.com.

${ }^{4}$ Head, Space Launcher Systems Analysis, martin.Sippel@dlr.de

${ }^{5}$ Project coordinator, Aerothermodynamics and Propulsion Analysis Section, rafael.molina@esa.int 


\section{Introduction}

$\mathrm{T}$ he European Commission (EC) co-funded project FAST20XX (Future High-Altitude High-Speed Transport 20XX) aims at exploring the frontier between aviation and space by investigating suborbital vehicles. The main focus is the identification and mastering of critical technologies for such vehicles rather than the vehicle development itself. The technology development is based on two advanced suborbital high-speed transportation concepts one for the medium term (5-10 years) and another for the longer term ( $>30$ years).

The long term concept is related to suborbital point-to-point long distance transport in very short time. Within this type of transportation, the SpaceLiner concept proposed by the DLR has been investigated ${ }^{1}$. It is a vertically launched two-stage rocket space vehicle system concept used to identify technologies required for suborbital ultrafast long-range transport of the long-term future.

One of the areas of investigation for such future transportation system is the flight control. At this stage the objective is not a detailed design of the Guidance, Navigation and Control (GNC) system, but to identify the requirements and constraints that will build the specification for such vehicles. The specification of the GNC has been built around the two flight mechanics domains: Flying Qualities and Flight Performance.

Flying Qualities analyses have been conducted to determine the trim, stability and control characteristics. The objective is to provide an early feedback to the aerodynamic team about the flying characteristics of the vehicle to support future evolutions of the concept. The design margins have been identified and a flyable angle of attack corridor is proposed. The aerodynamics of the vehicle has been verified and extended through Computational Fluid Dynamics calculations (CFD).

Regarding to Flight Performance, the entry corridor provides the margins in terms of trajectory control that are available for the guidance system. Guidance capability assessments have been conducted to determine the feasibility of the mission reference trajectory and to indentify alternative guidance strategies.

\section{The SpaceLiner Concept}

Since 2005, the Space Launcher Systems Analysis group (SART) of the German Aerospace Center DLR has been working on a novel vehicle concept for long range hypersonic passenger transport. The "SpaceLiner" is a large, rocket propelled vehicle (length $\sim 60 \mathrm{~m}$ ) that is launched vertically with launch and ascent being assisted by a reusable booster. Both, the hypersonic passenger stage and the booster utilize liquid propellants.

In contrast to other hypersonic vehicle concepts the SpaceLiner does not incorporate radically new or unproven technologies. Instead, rather conventional rocket propulsion systems and vertical ascent trajectories are used. Thus, the development of such a vehicle is more a political and financial question, and to a lesser extend a technological one. Nevertheless the SpaceLiner concept contains several technical and logistical challenges, such as active cooling technologies, passenger accommodation and safety together with the provision for suitable launch and landing sites.

The final objective of the SpaceLiner development is to dramatically reduce intercontinental travel times compared to today's subsonic passenger aircraft flights by travelling at hypersonic velocities. For example, a trip from Europe to Australia, which is the current reference mission, will last only 90 minutes. Also other intercontinental routes such as New York to Australia have been considered.

Several SpaceLiner concepts have been produced. This paper concentrates in the socalled SpaceLiner 4 concept (SL4).

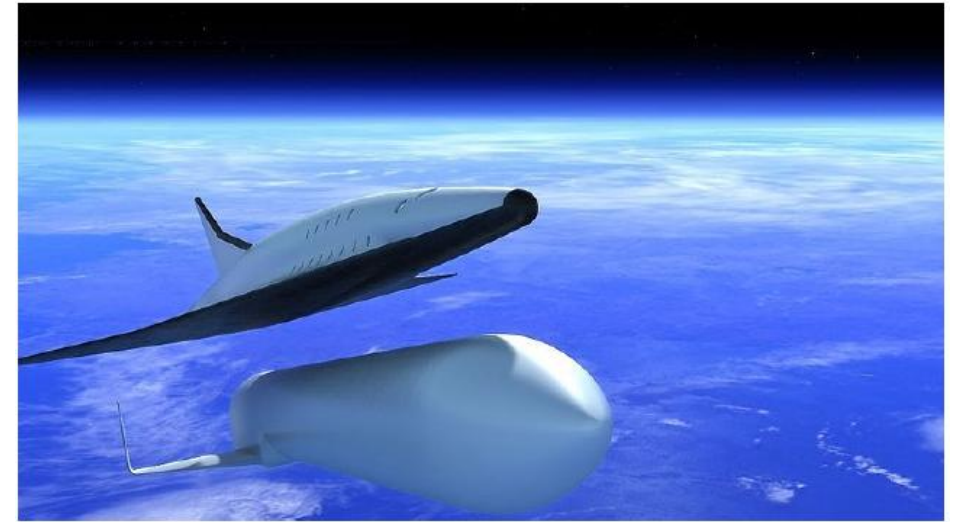

Figure 1. Artist's impression of the SpaceLiner2 at booster separation. 


\section{Aerodynamics}

An initial aerodynamics of the Spaceliner 4 concept was available for the conceptual design of the vehicle. As long as it is a key input that strongly drives the Flight Mechanics and GNC performance of the vehicle, a verification and extension of the dataset through detailed CFD analyses has been conducted.

The software used to run the CFD simulations is an in-house code based on: Euler flow modeling with tetrahedral spatial discretization; adaptive Mesh Refinement, feature-based (Mach number has been implemented for this work) involving anisotropic remeshing and mesh movement/adaptation; node Centered, blended anisotropic second- and fourth-order dissipation; local time stepping.

A total of 36 CFD cases has been conducted covering transonic to hypersonic regime and with and without elevator deflection (see Table 1). Not all Mach, AoA and elevator combinations have been inspected, but those of interest according to the flight envelope and initial trim assessments. The CFD representation of the vehicle includes a highly detailed surface mesh in which all geometrical aspects of the SpaceLiner CAD geometry have been modeled. Body flaps and wing elevator have been considered.

The results show that the initial aerodynamics was appropriate in the hypersonic regime, but refinement was needed in the supersonic and transonic part. A comparison The CFD results have been used to populate the aerodynamic dataset. Figure 1 shows a comparison of the longitudinal aerodynamics (lift, drag, pitch moment and L/D) between the conceptual aerodynamic dataset, which is based on fast engineering methods, and the CFD results.

An application rule has been created to calculate the aerodynamic coefficient as well as the stability and control derivatives needed for Flying Qualities:

$$
\mathrm{C}=\left(\mathrm{C} 0(\alpha, \mathrm{M})+\mathrm{CDE}\left(\alpha, \mathrm{M}, \delta_{\mathrm{e}}\right)+\mathrm{CDBF}\left(\alpha, \mathrm{M}, \delta_{\mathrm{bf}}\right)\right)(1+\mathrm{UC}(\mathrm{M}))
$$

Where $\mathrm{C} 0$ is the coefficient in clean configuration (no control surfaces deflection) and CDE and CDBF are the contribution of the wing elevator and body flap. Uncertainty is modeled as a scale factor dependent on Mach.

Table 1. CFD matrix of cases.

\begin{tabular}{ccc}
\hline Mach & AoA & Elevator \\
\hline 1.1 to 19.8 & 0 to $10^{\circ}$ & $-5^{\circ}$ to $5^{\circ}$ \\
\hline
\end{tabular}

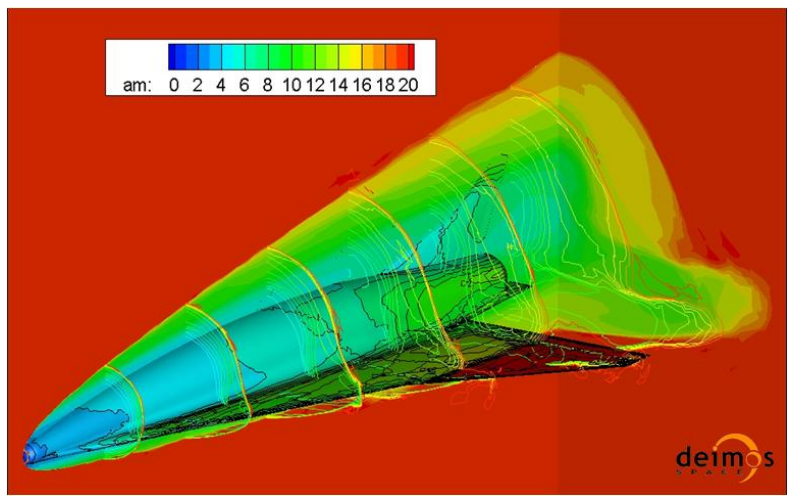

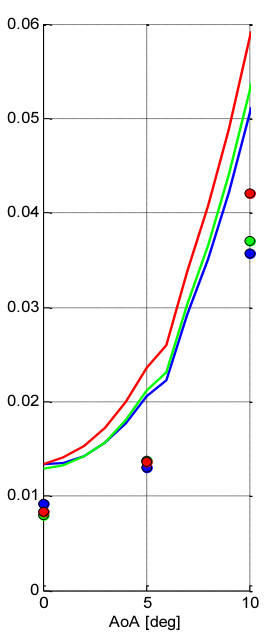

Figure 2. Comparison aerodynamics and CFD at Mach $10(\mathrm{CD}, \mathrm{Cm}$ and $\mathrm{L} / \mathrm{D})$
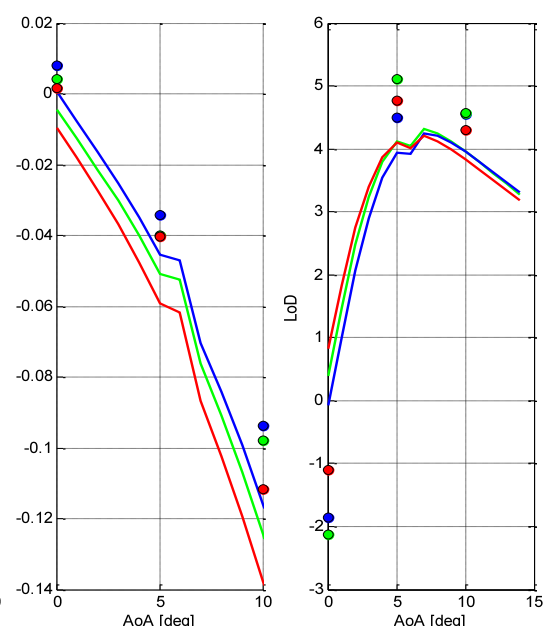

AoA [deg]

the

conceptual

Figure 1. Mach distribution at Mach 19.8

\section{Caerodynamics and CFD at Mach 10 (CD, Cmand $\mathrm{C} / \mathrm{D})$}

\section{Flying Qualities}

According to the Space Vehicles classification for Flying Qualities (FQ) proposed in Ref. 2, the SpaceLiner vehicle is of Class III, space plane, which comprises winged vehicles that generate aerodynamic lift through its body and wings and whose maneuverability exceeds that of lifting bodies (Class II) and capsules (Class I). The SpaceLiner flight covers hypersonic entry flight, descent and approach and landing into runway. Therefore, categories A, B and C apply.

In a hypersonic vehicle the angle of attack profile is strongly linked with the mission feasibility and hence its selection and assessment cannot be uncoupled from the trajectory design. The objective is the evaluation of the Centre of Gravity $(\mathrm{CoG})$ box provided by system team and the identification of the associated entry corridor. 
This process provides the range for the design of the nominal angle of attack (AoA) profile during the entry and identifies the available entry corridor for trajectory design.

The Flying Qualities analyses are performed with the Flying Qualities Analysis (FQA) Tool. This FQA Tool will enable a Flight Mechanics engineer to follow the steps that build the FQA Framework to successfully perform the required flight qualities analyses within a given program. The FQA Tool software package has the flexibility to connect to different vehicles models (e.g. capsule, space plane and lifting body) and data. The FQA Tool performs computations based on the user's inputs (mainly: vehicle models, flying quality objectives) in order to derive the criteria allowing the characterization of the flying quality of the vehicle and the definition of guidelines for the design of the GNC for the atmospheric reentry.

The system context makes reference to operational context in which the system or the simulator will operate, giving indication of its interaction with the environment in terms of inputs and outputs. Figure 3 presents the context use of the FQA Tool. The user of the FQA Tool will be an experienced flight mechanics analyst. It will use the FQA Tool to derive the criteria allowing the characterization of the flying quality of the vehicle and the definition of guidelines for the design of the GNC for atmospheric reentry. The FQA Tool can also interface with an external Worst Case Analysis Tool for the identification of worstcase combinations of dispersions for the flying quality analysis.

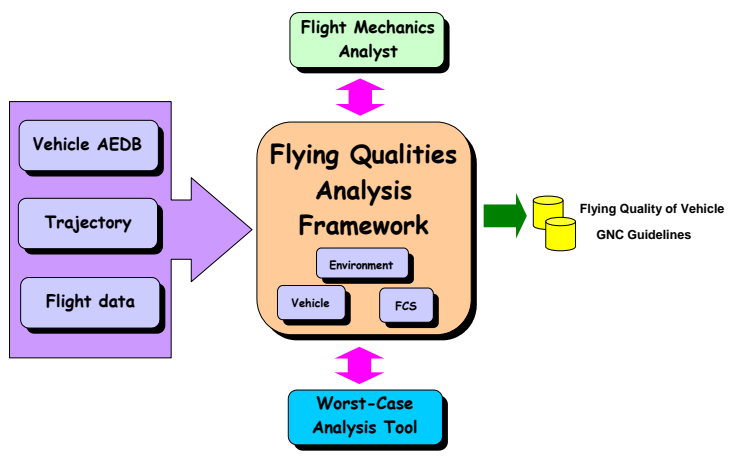

The Angle of Attack corridor for the Spaceliner 4 concept using the conceptual aerodynamic dataset and taking into account uncertainties in the aerodynamic dataset is presented in Figure 1. It represents the domain where the angle of attack can be optimized respecting the Flying Qualities constraints for the vehicle. These results correspond to the Moment Reference Centre (MRC) of the vehicle.

In nominal conditions, for the nominal $\mathrm{CoG}$, it is possible to design a trim line that respects all the constraints. However, dispersions on AEDB and CoG strongly affect the corridor and it is not possible to respect all the constraint at all the flight regimes in dispersed conditions. From a system perspective that means that CoG relocation is needed, as well as a review of the aerodynamic layout in order to avoid the complexity of a flight control system handling an unstable vehicle.

One of the candidate reference trajectories for the SL4 concept was assessed from a FQ perspective for the gliding reentry phase (Figure 1). The angle of attack profile was generated independently from the AoA corridor considerations and therefore non-compliance of the stability limits is expected. The objective of the Flying Qualities campaign is to evaluate in detail the trim and stability characteristics taking into account uncertainties mainly in the aerodynamics and mass, $\mathrm{CoG}$ and Inertia properties. Table 2 summarises the uncertainties being considered, which includes a $\mathrm{CoG}$ box around the reference $\mathrm{CoG}$ location and dispersion in the AoA to cope with navigation uncertainties and control dispersion around the trimmed value. Uniform dispersion is considered in the aerodynamics to cope with the large uncertainties associated to a preliminary aerodynamic database.
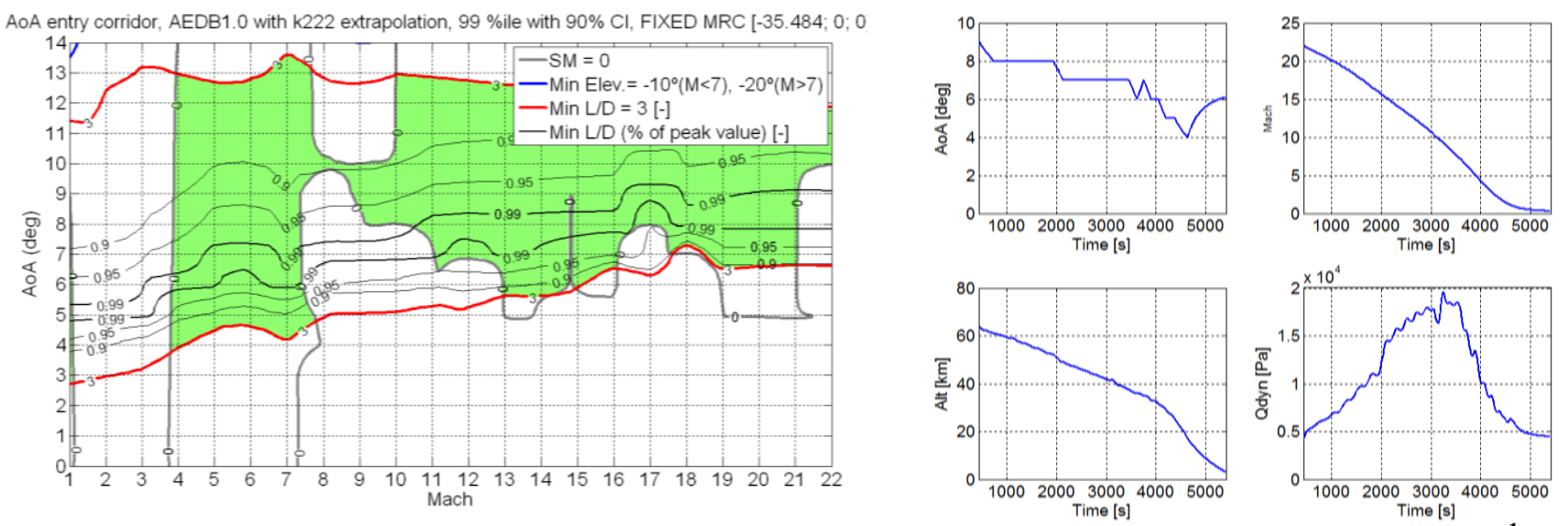

Figure 4. Angle of Attack corridor with dispersed AEDB at Figure 5. SpaceLiner 4 Reference trajectory ${ }^{1}$. MRC 
Table 2. uncertainties for the Flying Qualities assessment

\begin{tabular}{llll}
\hline Item & Uncertainty & Type & Units \\
\hline Aerodynamics & $+/-1$ & Uniform & - \\
AoA trim & AoA $+/-2$ & Uniform & $\mathrm{deg}$ \\
CoG location $-\mathrm{X}$ & $\mathrm{xG}+/ 1$ & Uniform & $\mathrm{m}$ \\
CoG location $-\mathrm{Y}$ & $\mathrm{yG}+/-0$ & Uniform & $\mathrm{m}$ \\
CoG location $-\mathrm{Z}$ & $\mathrm{zG}+/-0.1$ & Uniform & $\mathrm{m}$ \\
$\begin{array}{l}\text { Roll, Pitch, Yaw inertia } \\
\text { moment }\end{array}$ & {$[\mathrm{Ixx}$, Iyy, Izz $]+/-10 \%$} & Uniform & $\mathrm{kg} . \mathrm{m} 2$ \\
\hline
\end{tabular}

A MonteCarlo campaign of 1000 shots for the Flying Qualities has been run with these uncertainties and the $99 \%$ rage of variability of the main stability and control indicators has been evaluated. The trim capability for the proposed $\mathrm{CoG}$ is shown in Figure 6. No trim problems are foreseen and deflections remain within a moderate range. The wing elevator sizing is therefore confirmed. More backward location would be favourable to reach a better balance between hypersonics and supersonics ranges with more positive deflections at low speed.

In terms of stability, Figure 7 confirms the longitudinal instability of the concept for the proposed centre of gravity. Therefore, a forward CoG relocation is required to improve stability or a revisit of the wing shape and location. These considerations have been taken into account in further evolutions of the concept.

A large L/D is exhibited by the vehicle, with a significant variability, which may compromise the vehicle range capability in adverse conditions. It is addressed in next section.

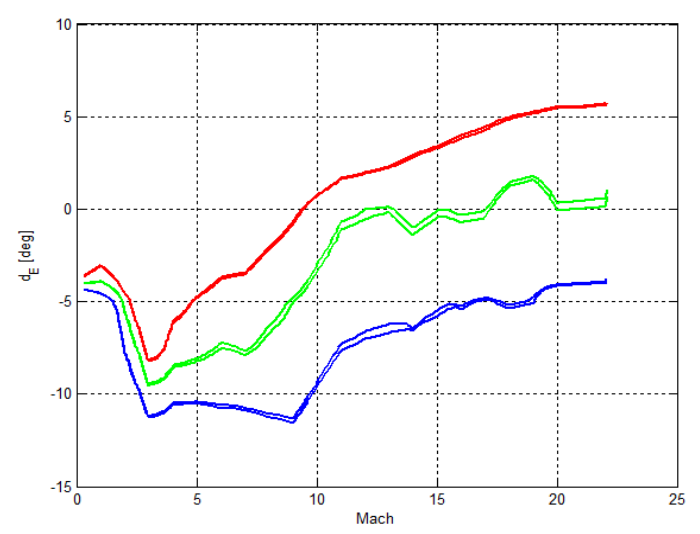

Figure 7. FQ: elevator variability $(99 \%$ range with $90 \%$ confidence level)
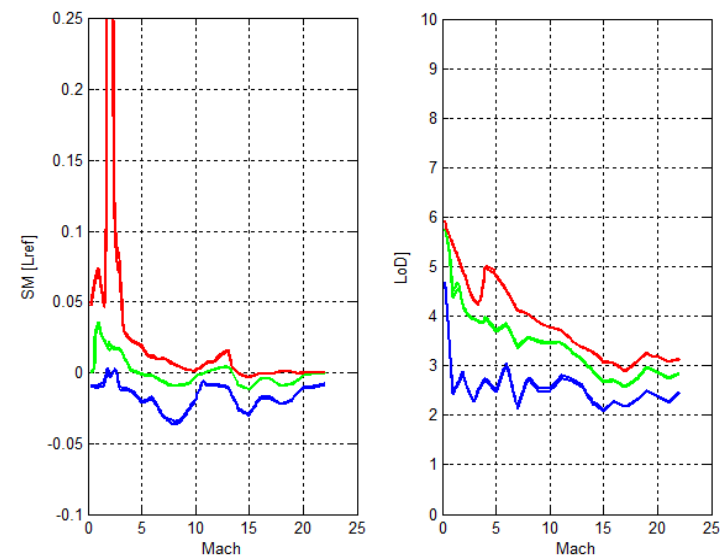

Figure 6. FQ: Static Margin and L/D variability (99\% range with $90 \%$ confidence level)

\section{Guidance Concept}

The guidance problem for a planetary entry vehicle is constituted by three main features: entry strategy, guidance technique and guidance method

The entry strategy defines how the hypersonic entry should be performed. Several solutions exist highly associated to the vehicle aerodynamic capability and the mission and system requirements: both direct entries (long and short) or indirect entries (skip and multi-skip) are available.

Given the high efficiency of the SpaceLiner 4 (SL4) vehicle and the characteristics of the reference trajectory the adopted solution is a long direct entry, in which the vehicle takes advantage of its high lift capability to maintain high altitude and high speed to increase the range flown.

The guidance technique defines the form to fly within the entry corridor. For the SL4 reference mission, a freeprofile technique is implemented in order to match the reference trajectory profiles coming from optimisation. Moreover, in case of on-board replanning needs, a direct design of the flyable trajectory within the entry corridor using a fixed shape technique is preferred.

Finally, the guidance method refers to the actual trajectory controller. It generates the proper guidance commands in order to fly the reference trajectory. Considering the SL4 mission characteristics a candidate trajectory tracker method has been selected to be implemented for assessing the guidance capability of the vehicle. The method represents an evolved solution based on an adaptive drag tracking controller.

The assessment of the guidance capability of the vehicle to fly the nominal trajectory in the presence of uncertainties and perturbations constitute the basis of the performance evaluation of the vehicle during the entry phase. The proposed algorithm is a drag-tracking scheme that considers both Trajectory generation (on-board trajectory planning) and Trajectory control (trajectory tracking). The commanded values are the bank angle and the angle of attack if active control surfaces are available. It is an evolution of the classic drag-velocity tracking algorithms used in Apollo/Shuttle/ARD/IXV and proposed for many other vehicles (Hermes, X38...). 
The reasons behind the selection of this guidance algorithm are: easy adaptability to the candidate vehicles, no restriction regarding to the reference trajectory shape, adaptive control, high order dynamics, energy based onboard trajectory re-planning and an analytical form that ensures a low CPU loading of the on-board GNC computer. The trajectory in controlled mainly though bank modulation in order to keep the angle of attack under strict limits in the hypersonic phase, where thermal loads are relevant and any AoA excursion can lead to a collapse of the system.

An Angle of Attack modulation with more authority is suggested for the low supersonic flight during the heading alignment phase.

\section{Guidance assessments}

A Monte-Carlo campaign has been performed using detailed environment models (atmosphere, winds, gravitational harmonics...) and uncertainties into the initial state, the vehicle (aerodynamics and mass properties) and environment (atmosphere and winds).

A reference trajectory has been computed in Ref.1 as a proof of concept of the SpaceLiner vehicle and Mission. However, the analysis of the guidance capability in nominal conditions has shown two characteristics of the reference trajectory that limit the trajectory control capability. First, the proposed reference angle of attack maximized the lift-to-drag ratio, which means that angle of attack authority to increase the L/D in case of a short range dispersed flight is not feasible. Second, the bank angle all along the flight is low, which besides the maximum L/D maximized the vehicle range but that provides a small margin for trajectory retargeting.

Figure 5 shows the guided trajectory in nominal conditions (no dispersions), showing the reduced margins with respect to the ceiling limit.

In case of uncertainties, this lack of margins is revealed. Figure 2 shows that the guidance scheme is able to keep the trajectories within the entry corridor and good tracking performances are achieved. However, targeting of the Terminal Area Energy Management Conditions at Mach 0.3 fails and large deviation are observed (Figure 1), mainly caused by the lateral guidance logic as there is no bank margin allocated by the reference trajectory to ensure crossrange manoeuvring.

In order to solve ensure good lateral controllability, an alternative entry trajectory has been proposed in order to increase the bank angle margins. For the same Main Engine Cut-Off (MECO) condition, the vehicle flies with an increased bank of the order of $40^{\circ}$.

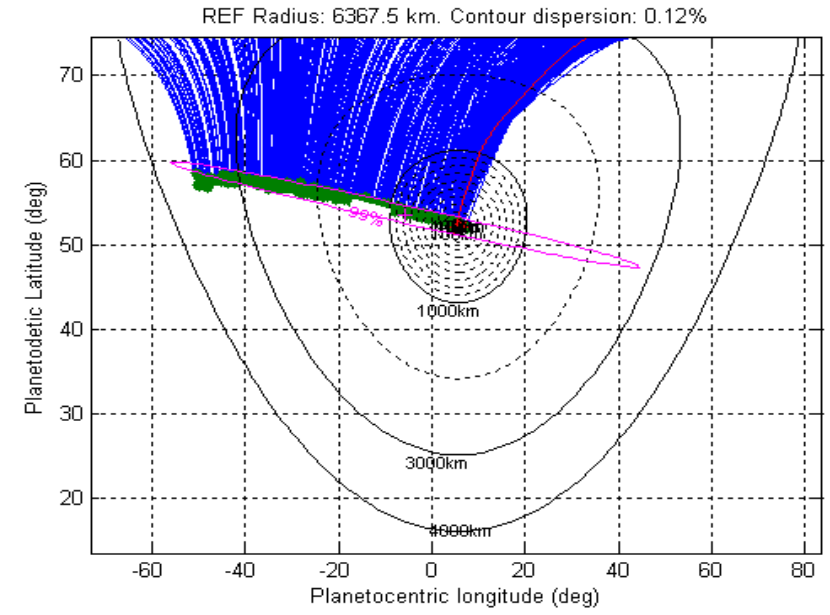

Figure 9 Monte Carlo campaign: dispersion at Mach 0.3 gate for the failed scenario

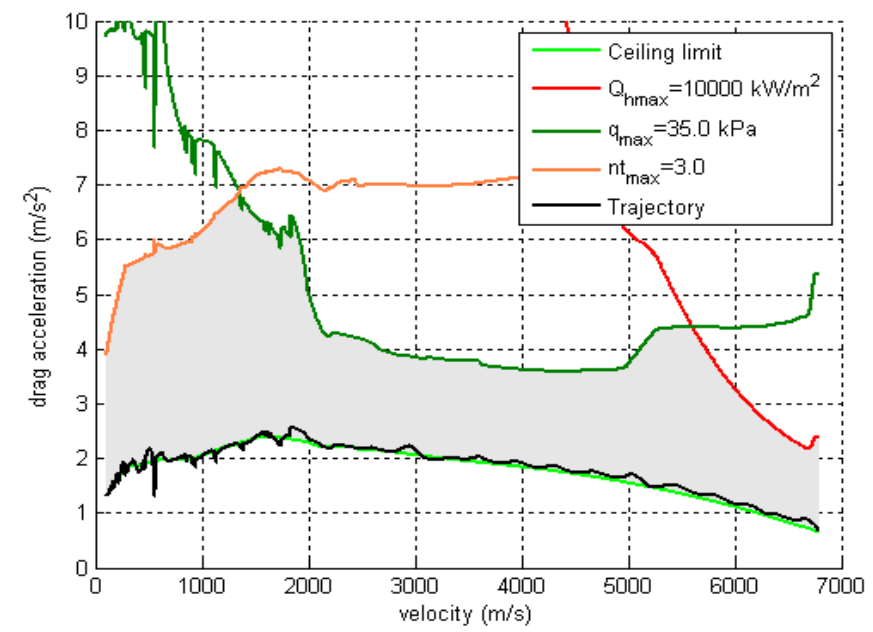

Figure 8 nominal trajectory within the entry corridor.

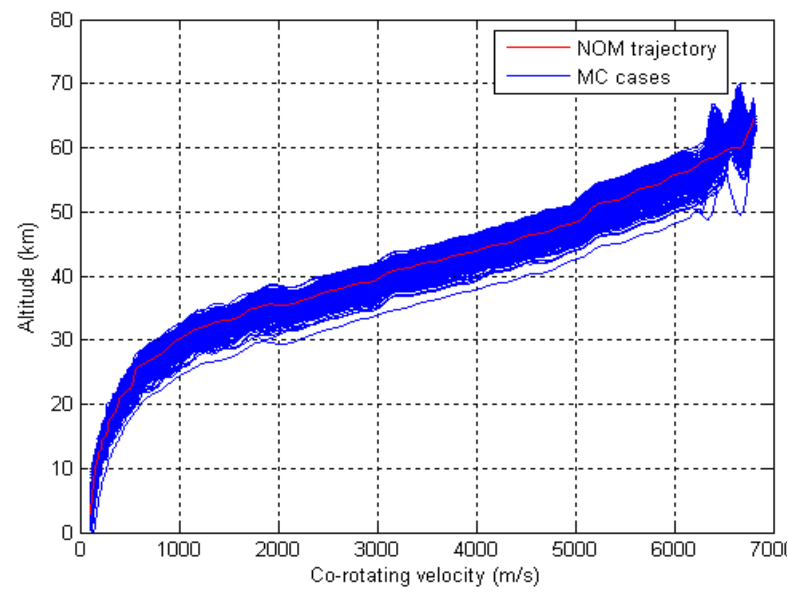

Figure 10 Monte Carlo campaign: guided trajectories in the altitude - velocity plane. 
The on-board replanner automatically modifies the ground track in order to target the final approach conditions (Figure 12). The same angle of attack profile is applied in order to not to modify the aerodynamics and aerothermodynamic behaviour. The performances significantly improves with a reduced number of failed cases but basically keeping position dispersion at Mach 0.3 in the order of $15 \mathrm{~km}$ without a fine tuning of the guidance gains. Despite the increase of the bank angle, the load factor is still within the requirements (Figure 11).

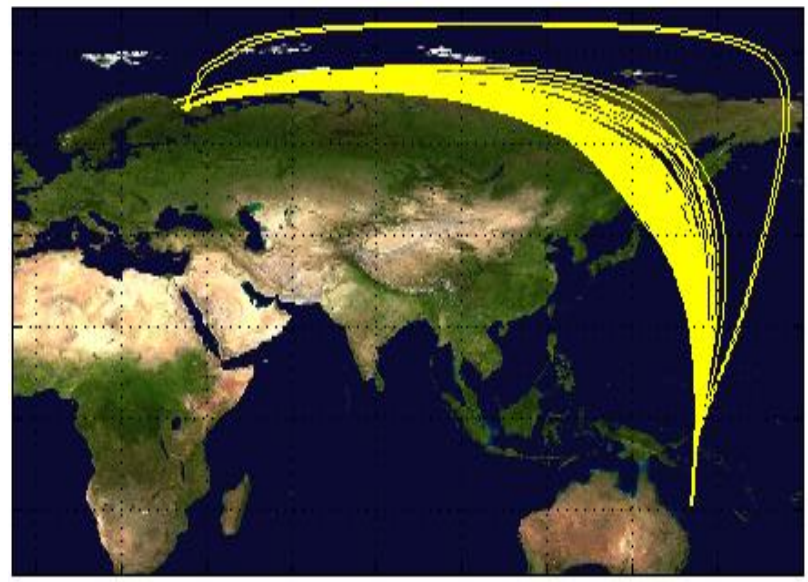

Figure 12 ground track of dispersed guided trajectories for the alternative entry trajectory.

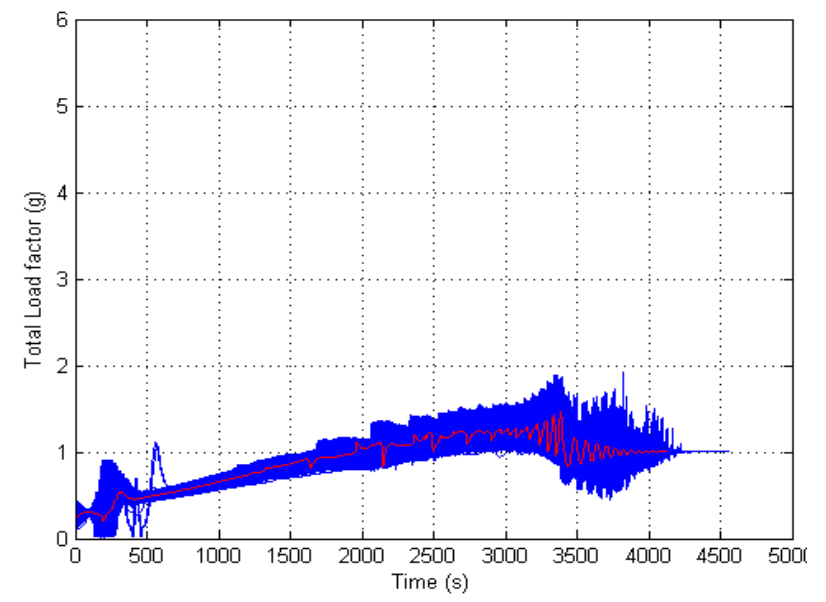

Figure 11 load factor profiles from Monte Carlo campaign.

\section{Conclusion}

In the frame of the European Commission (EC) co-funded project FAST20XX (Future High-Altitude HighSpeed Transport 20XX) an analysis of the Guidance, Navigation and Control characteristics for the Spaceliner concept 4 has been conducted in order to derive the trajectory and attitude control requirements for a hypersonic long range transportation concept. The conclusions and recommendations have been used to support the development of later SpaceLiner concepts. This paper focuses on the gliding part of the flight once the booster engine has been separated.

The aerodynamics of the vehicle is one of the main drivers of the fight mechanics and GNC analyses and hence validation and confirmation since the first steps is required in order to avoid not reliable feasibility conclusions. A detailed CFD campaign has been conducted to verify the applicability of the conceptual design dataset and has been used as basis for the consolidated design dataset.

The Flying Qualities analyses for the SpaceLiner concept show that a flyable corridor exist, but stability margins are reduced or even non-existing in some regions of the flight envelope. Therefore, mode detailed CoG location analyses together with the system team are suggested to derive a vehicle configuration leading to a feasible flight mechanics concept as well as a revisit of the wing shape and location. The Flying Qualities assessment constitutes the source of specification for the GNC and advances the flight control performance and issues. For the candidate reference trajectory, the Flying Qualities have been evaluated in detail. The SL4 concept shows adequate longitudinal trim characteristics, while longitudinal instability, in accordance with AoA corridor analyses, is observed all along the flight.

Adequate margins both in terms of angle of attack and bank angle are required for a gliding vehicle in which entry corridor control and range control is performed through drag adjustments. The proposed reference trajectory does not provide enough margins to ensure right longitudinal and lateral targeting of the TAEM conditions in subsonics. An alternative feasible entry trajectory has been identified providing margins for the trajectory control function and leading to performances compliant with the requirements. The guidance assessments have shown the compatibility of the concept with guidance concepts typical for re-entry vehicles based on drag tracking and bank modulation. 


\section{References}

${ }^{1}$ Sippel, M., van Foreest, A., Bauer C., Cremaschi F., "System Investigations of the SpaceLiner Concept in FAST20XX" 17th AIAA International Space Planes and Hypersonic Systems and Technologies Conference, 11.-14. April 2011, San Francisco, CA, USA.

${ }^{2}$ Haya-Ramos, R. at al. "Flying Qualities Analysis for Re-entry Vehicles: Methodology and Application”. AIAA Guidance, Navigation, and Control Conference 2011, Portland, USA 